\title{
Pratiques d'élevage extensif et performances de bovins de race locale, et croisée avec des races laitières exotiques en République démocratique du Congo
}

\author{
D.K. Kibwana ${ }^{1,3}$ A.M. Makumyaviri ${ }^{1,2}$ J.L. Hornick ${ }^{3 *}$
}

\begin{abstract}
Mots-clés
Bovin - Race d'animal d'élevage Croisement - Performance animale Méthode d'élevage - République démocratique du Congo.
\end{abstract}

\begin{abstract}
Résumé
Afin d'analyser les pratiques d'élevage et leurs relations avec les performances zootechniques de bovins de race mixte, lait et viande, en territoire de Beni, province du Nord-Kivu, République démocratique du Congo, une étude a été menée de juillet 2003 à novembre 2006, sur 8676 bovins, provenant de 65 élevages et comportant 4945 vaches de race locale, et croisées avec des races exogènes considérées amélioratrices. Des enquêtes, des observations démographiques, des mesures de production laitière et des suivis de reproduction ont été effectués dans les exploitations de l'étude. Les données ont été analysées à l'aide du test du chi carré et de modèles linéaires généralisés, en fonction du type génétique, de la qualité du fourrage et du type de complément. Les résultats ont montré un certain niveau de technicité des éleveurs, un faible renouvellement des populations bovines et une influence des pratiques d'élevage sur la production laitière, l'âge au premier vêlage et l'intervalle entre vêlages. Chez les vaches de type croisé, la production laitière journalière a été plus du double de celle observée chez la race locale. La complémentation sous forme de fourrages et de concentrés a permis d'augmenter significativement mais faiblement la production laitière par rapport à la situation sans complément. L'âge au premier vêlage et l'intervalle entre vêlages ont été influencés par le type génétique, et l'intervalle entre vêlages également par la complémentation et le type de fourrage. Ces résultats montrent l'importance de la conduite des élevages sur les performances laitières et reproductives des vaches dans la région d'étude.
\end{abstract}

\section{INTRODUCTION}

Dans les régions tropicales humides d'altitude de l'Afrique de l'Est, les systèmes d'élevage bovin sont extensifs ou semi-intensifs (24), si les surfaces pâturables sont suffisamment importantes compte tenu de la densité de la population humaine. Les races bovines locales qui y sont exploitées sont adaptées à leur milieu

\footnotetext{
1. Faculté de médecine vétérinaire, Université catholique du Graben, République démocratique du Congo.

2. Faculté de médecine vétérinaire, Université de Lubumbashi, Lubumbashi, République démocratique du Congo.

3. Faculté de médecine vétérinaire, service de Nutrition des animaux domestiques, Université de Liège, boulevard de Colonster 20, bât. B43, 4000 Liège, Belgique.

*Auteur pour la correspondance

Tél. : +32 (0) 43664131 ; fax: +32 (0) 43664122

E-mail : jlhornick@ulg.ac.be
}

mais manifestent de faibles performances laitières (12). Elles sont soumises à un niveau de complémentation limité ou inexistant (8), et à une gestion médiocre de la reproduction. Les éleveurs tentent alors de rémédier à ces faiblesses en pratiquant des croisements avec des taurins exotiques à meilleur potentiel laitier (6). Bien que l'introduction des bovins de races européennes (Pie-noire Hollandaise, Brun-Suisse, Jersey) et asiatiques (Sahiwal) à performance laitière reconnue date de la fin du $\mathrm{XIX}^{\mathrm{e}}$ siècle, les données sur le niveau génétique des animaux ne sont pas disponibles dans la zone de la présente étude. Ces croisements sont voués à l'échec sans bonnes pratiques d'élevage et notamment sans une complémentation adéquate à l'aide de cultures fourragères ou de concentrés (29). Plusieurs études réalisées en milieux tropicaux ont en effet montré que l'alimentation et le croisement sont des facteurs clefs de l'amélioration de l'élevage (11). Dans une région donnée, l'établissement d'un diagnostic relatif aux pratiques d'élevage est donc un prérequis indispensable à tout projet d'amélioration. 
A la connaissance des auteurs, les pratiques d'élevage dans le territoire de Beni, au Nord-Kivu, République démocratique du Congo, sont faiblement documentées. Il a été rapporté que l'élevage extensif des bovins est largement répandu dans la province du NordKivu et qu'il est caractérisé par des effectifs de plus ou moins 30 à 50 têtes par troupeau (18). Mais aucune étude montrant les effets des pratiques d'élevage sur les performances des animaux n'a été publiée à ce jour.

La présente étude a ainsi eu pour objectif de caractériser les pratiques d'élevage adoptées par les éleveurs de bovins en territoire de Beni et de les mettre en relation avec les niveaux de production laitière et les paramètres de reproduction des animaux.

\section{MATERIEL ET METHODES}

L'étude a été conduite de juillet 2003 à novembre 2006 dans le territoire de Beni (figure 1), couvrant une superficie de $7484 \mathrm{~km}^{2}$ et occupé par une population estimée à 879200 habitants. Il est situé dans la zone équatoriale sous un climat tropical humide à une altitude moyenne de 1500 mètres environ. La pluviométrie moyenne est de $1716 \mathrm{~mm} / \mathrm{an}$ avec des températures maximales de septembre à octobre et de mars à avril. Les précipitations mensuelles les plus faibles sont enregistrées entre janvier et février, et entre juillet et août. La température moyenne varie entre $23^{\circ} \mathrm{C}$ (au nord) et $25^{\circ} \mathrm{C}$ (dans le secteur forestier).

Parmi les 109 fermes enregistrées par le service de l'Agriculture du territoire, 65 ont été retenues sur la base de la disponibilité des éleveurs. Les données ont été obtenues à partir de plusieurs sources : (a) des enquêtes effectuées sur la base d'un questionnaire visant à déterminer, à l'aide de questions ouvertes ou fermées, le type de complémentation utilisé et l'existence éventuelle de cultures fourragères, l'âge estimé des animaux, l'âge au sevrage, l'âge au premier vêlage, l'intervalle entre vêlages, le nombre et les catégories d'animaux vendus, la destination des résidus de récolte, le suivi ou non de l'exploitation par des cadres vétérinaires ; (b) des observations directes et/ou des consultations de documents administratifs tenus par les éleveurs, intéressant tous les animaux présents sur l'exploitation, soit l'âge déterminé par l'examen de la dentition et des cornes, le sexe, la race, la composition floristique des pâturages ; et (c) des observations et des suivis limités aux vaches laitières et se rapportant aux principaux paramètres zootechniques déterminant la rentabilité de l'exploitation, soit l'âge au premier vêlage, l'intervalle entre vêlages, le taux de fertilité, la production laitière, le taux de mortalité. Le stade et le rang de lactation ont été relevés lors d'entretiens et par l'exploitation des documents administratifs tenus par les fermiers.

Les mesures et les suivis ont été effectués par dix cadres vétérinaires supervisant chacun en moyenne six fermes se trouvant dans le même secteur. Le passage des membres de l'équipe chargés de la récolte des données a été effectué deux fois toutes les deux semaines dans chaque ferme, le reste de temps était passé dans les autres exploitations du même secteur. La continuité de la récolte des données relatives à la production laitière a été menée par des bouviers alphabétisés, instruits par les enquêteurs et assistés par le fermier, le matériel de récolte de lait et de poids des veaux ayant été remis à des éleveurs qui n'en disposaient pas.

Les exploitations ont ensuite été classées en type I, ayant exclusivement recours aux pâturages naturels, et en type II, disposant de cultures fourragères. La supplémentation a été définie par son absence (A), par la distribution de blocs à lécher et/ou de sel gemme (S), et par la distribution plus élaborée (E) de compléments, associés ou non aux compléments de type $\mathrm{S}$. Les vaches en lactation ont été réparties, selon leur phénotype, en animaux de race locale (L) et métis (type croisé, $\mathrm{C}$ ).

La traite des vaches a été effectuée manuellement dans des récipients gradués par les bouviers, en présence de membres de l'équipe des enquêteurs, deux fois par jour, à 8 heures et à 17 heures, le veau amorçant la descente du lait par une courte tétée. Les quantités moyennes de lait par animal, par jour (matin et soir) et par phénotype (tous les mois et tous les rangs de lactation confondus) ont été obtenues sur une traite complète de trois quartiers de la mamelle à laquelle a été ajoutée celle du quartier réservé au veau obtenue par pesée avant et après la tétée. Elles ont permis de calculer la production laitière moyenne de chaque troupeau sur la période d'enquête et de suivi, tous stades et tous rangs de lactation confondus. Ces quantités ont été régulièrement enregistrées par les enquêteurs et les agents de la ferme.

Les données catégorielles ont été analysées par le test du chi carré et les données continues à l'aide de modèles linéaires généralisés (GLM, Minitab). L'unité expérimentale a été le sous-troupeau, de type génétique $\mathrm{L}$ ou $\mathrm{C}$, dans une ferme. L'étude des effets du type génétique, du type de fourrage et du type de complément a été réalisée à l'aide du modèle suivant :

$\mathrm{Y}_{\mathrm{ijkl}}=\mu+\mathrm{a}_{\mathrm{i}}+\mathrm{b}_{\mathrm{j}}+\mathrm{c}_{\mathrm{k}}+\mathrm{ab}_{\mathrm{ij}}+\mathrm{ac}_{\mathrm{ik}}+\mathrm{e}_{\mathrm{ijk} \mathrm{l}}$

où $\mathrm{Y}_{\mathrm{ijkl}}$ est la production laitière journalière, $\mu$ la moyenne générale, $a_{i}$ l'effet du génotype $i(L$ ou $C), b_{j}$ l'effet du type du complément $j$ (A, S ou E), $c_{k}$ l'effet du type de fourrage $k$ (I ou II), ab ${ }_{i j}$ et $a c_{i k}$

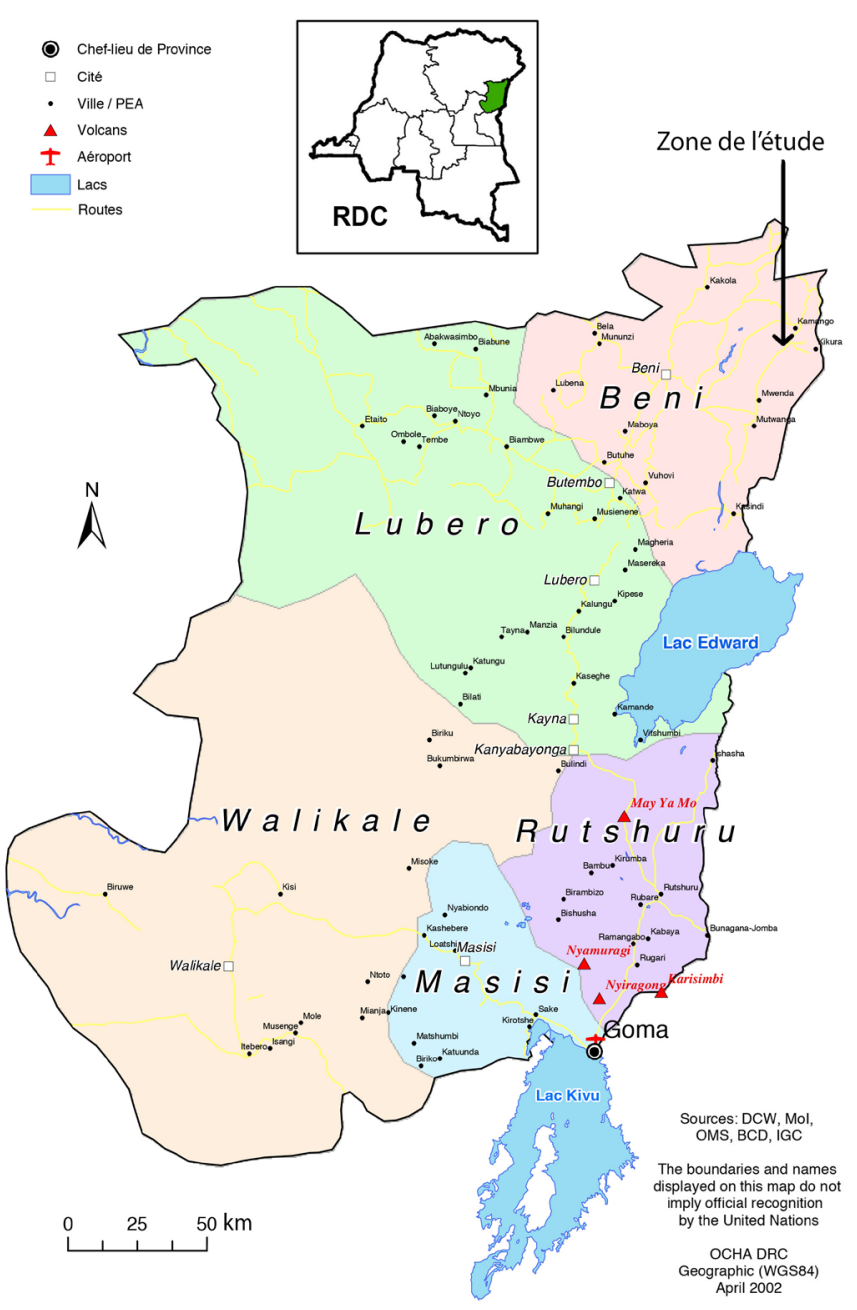

Figure 1 : carte des territoires du Nord-Kivu, RDC. 
sont les interactions génotype $\mathrm{x}$ type de complément et génotype $\mathrm{x}$ type de fourrage, et $\mathrm{e}_{\mathrm{ijkl}}$ est l'effet résiduel aléatoire. Le modèle de sommes de carrés de type 3 (SS3) a été retenu, supposant le maintien de l'orthogonalité des données. Une pondération basée sur les effectifs des sous-troupeaux a été appliquée au modèle.

\section{RESULTATS}

Les recensements ont pu être effectués sur 8676 bovins et les performances zootechniques sur 4945 vaches en lactation. La majorité des éleveurs pratiquait l'élevage seul, le reste combinait l'élevage à l'agriculture de subsistance, sans aucun recyclage organisé des sous-produits des cultures vivrières dans l'alimentation des animaux. Les ressources fourragères étaient des pâturages de type naturel (type I) composés essentiellement de Pennisetum clandestinum, Brachiaria sp., Paspalum sp., Panicum maximum, Trifolium pratense, avec une proportion de 25 p. 100 environ de légumineuses, notamment Desmodium sp. et Centrosema sp. D'autres graminées envahissantes ont été présentes, telles que Sporobolus pyramidalis et Imperata cylindrica. Les pâturages de bas-fonds marécageux ont été caractérisés par les Cypéracées, notamment Carex sp., Cyperus sp. Les exploitations de type II, outre les formations herbacées ci-dessus, ont également disposé de cultures fourragères destinées à la fauche, constituées de Tripsacum laxum, Setaria sp., Leucaena leucocephala et Stylosanthes guianensis. Seulement 25 p. 100 des éleveurs enquêtés ont eu recours aux cultures fourragères.

La supplémentation de type $\mathrm{S}$ a consisté en une simple distribution de blocs de sel gemme et/ou des blocs à lécher de compositions variables laissés à volonté aux animaux, et celle de type $\mathrm{E}$ en une distribution plus élaborée de compléments à base de tourteau de palmiste ou d'arachide et de son de diverses origines (riz et blé), associé ou non aux compléments de type $\mathrm{S}$. Les compléments alimentaires n'ont été distribués qu'aux vaches du début à la fin de lactation. Une minorité d'éleveurs ne pratiquaient pas la complémentation (14 p. 100). Les autres étaient répartis en une moitié (44 p. 100) distribuant un simple bloc à lécher et l'autre (42 p. 100) apportant en plus des concentrés.

Près de 70 p. 100 des élevages étaient encadrés par un agent vétérinaire visitant l'exploitation à intervalles réguliers ou résidant dans l'exploitation même.

Les croisements étaient réalisés à partir d'un taureau croisé introduit dans le troupeau. Seuls 4,6 p. 100 des éleveurs pratiquaient l'insémination artificielle à partir des semences exotiques pures citées plus haut, importées de Kampala. Parmi les exploitations, 18,5 p. 100 ont eu recours à des mâles reproducteurs provenant d'autres exploitations. L'objectif principal des éleveurs était d'améliorer les performances laitières des races locales.

Les élevages enquêtés comprenaient majoritairement (89,2 p. 100) des animaux de race locale, notamment Ankole et Lugware, et des métis issus d'un croisement entre race locale et exogène, essentiellement de type Frison, Brun-Suisse, Jersey, ou Sahiwal. En outre, 9,2 p. 100 des élevages comportaient des animaux de race locale uniquement et 1,5 p. 100 des animaux de type croisé seul. L'effectif moyen des troupeaux a été de 133,5 $\pm 0,2$ bovins comprenant 78 p. 100 de femelles et composés en moyenne de 76,1 $\pm 0,1$ vaches de plus de 3 ans et de 2,3 $\pm 0,0$ taureaux. La structure des troupeaux est représentée sur la figure 2. Le ratio jeunes (0-3 ans) / adultes (> 3 ans) a différé selon la race, soit 81,3 p. 100 pour la race locale $v s 51,5$ p. 100 pour les métis $\left(\mathrm{c}^{2}=8,7 ; \mathrm{P}<0,05\right)$. Le ratio veaux (tous sexes et toutes races confondus) / vaches a été de 23,7 p. 100 vs 12,2 p. 100 pour le ratio sevrés pré-pubères (tous sexes et toutes races confondus) / vaches. Les vaches adultes, ainsi que les catégories de jeunes animaux (du veau au taurillon) ont représenté les lots les plus importants. Les mâles âgés de plus de trois ans (castrés ou entiers) ont représenté 3,3 p. 100 de la population totale. Les sous-troupeaux de type croisé ont comporté davantage d'adultes, en particulier des vaches (en moyenne 39 vaches de type croisé vs 37 vaches de race locale). La proportion de jeunes animaux a diminué avec l'âge, tandis que les jeunes animaux pubères, spécialement les génisses, ont eu des effectifs plus élevés. Près de 73 p. 100 des éleveurs sevraient les veaux à l'âge de huit mois, les autres le faisaient au-delà de cet âge.

Le taux de fertilité des vaches de race locale a été de 52,5 p. 100 vs 41,3 p. 100 chez les vaches croisées, bien que les naissances aient été réparties sur toute l'année et sur toute la période de l'étude. Les taux de mortalité de 0 à 1 an ont été de 4,5 p. 100 chez la race locale vs 6,4 p. 100 chez les animaux croisés, et de 2,0 et 4,2 p. 100 respectivement de 1 à 2 ans. Les mortalités de 0 à 2 ans ont été significativement influencées par la race : elles ont été près de deux fois plus importantes chez les animaux croisés $\left(c^{2}=5,7 ; \mathrm{P}=0,017\right)$.

Concernant les mouvements, 3,4 animaux (soit 2,6 p. 100) ont été vendus par an et par exploitation et 23 p. 100 des éleveurs ont acheté des animaux reproducteurs de remplacement, à raison de 1,3 animal par an en moyenne. Ces animaux consistaient en des génisses, des taurillons et des taureaux. Les données sur le nombre d'animaux intervenus dans le troc n'ont pas pu être accessibles.

Les effets du type génétique, de la complémentation et du type de fourrage sur les paramètres zootechniques sont indiqués dans le tableau I. La production laitière journalière a été comprise entre 2,4 $\pm 0,2$ et 5,2 $\pm 0,2 \mathrm{~L} /$ vache. Elle a été influencée significativement par le type génétique, le type de complément et le type de fourrage distribué $(\mathrm{P}<0,05$ à 0,001$)$. Elle a été plus du double dans le type croisé. La distribution de fourrage a augmenté la production moyenne d'un litre $(4,3 \pm 0,2 \mathrm{~L}$ vs $3,3 \pm 0,1 \mathrm{~L} ; \mathrm{P}<0,001)$, la complémentation de type élaboré l'ayant augmentée de 0,4 litre par rapport à la situation sans complément, et de 0,5 litre par rapport à une complémentation simple $(\mathrm{P}<0,05)$.

Les effets du type de fourrage et de la complémentation n'ont pas été significatifs sur l'âge au premier vêlage. Toutefois, ce paramètre a été fortement influencé par le type génétique $(38,4 \pm 0,8$ mois chez les vaches de race locale vs 29,7 $\pm 0,8$ mois chez les vaches de type croisé $; \mathrm{P}<0,001)$.

Tous les facteurs étudiés ont influencé l'intervalle entre vêlages de manière significative. Il a été de 21,8 $\pm 0,5$ mois chez les vaches

Femelles
Adultes 37 mois et plus
Pubères 24-36 mois
Sevrés prépubères 19-23 mois
Sevrés prépubères 10-18 mois

Figure 2 : pyramide des âges (\% moyen des catégories d'âge, par race) dans des élevages bovins à Beni (République démocratique du Congo) sur un total de 8676 animaux. 


\section{Tableau I}

Effets du type génétique, de la complémentation et du type de fourrage sur différents paramètres zootechniques (moyennes moindres carrés \pm écart-type) en élevage bovin à Beni, République démocratique du Congo

\begin{tabular}{|c|c|c|c|c|c|c|c|c|c|c|}
\hline \multirow[t]{2}{*}{ Paramètres } & \multicolumn{2}{|c|}{ Type ${ }^{1}$} & \multicolumn{3}{|c|}{ Complément $^{2}$} & \multicolumn{2}{|c|}{ Fourrage $^{3}$} & \multicolumn{3}{|c|}{$P>F$} \\
\hline & $\mathbf{L}$ & C & A & S & $\mathbf{E}$ & I & II & Type & Compl. & Fourr. \\
\hline \multicolumn{11}{|c|}{ Production laitière traite } \\
\hline (L/jour/vache) & $2,4 \pm 0,2$ & $5,2 \pm 0,2$ & $3,7 \pm 0,2 \mathrm{a}, \mathrm{b}$ & $3,6 \pm 0,2^{a}$ & $4,1 \pm 0,2^{b}$ & $3,3 \pm 0,1$ & $4,3 \pm 0,2$ & $* * *$ & * & $* * *$ \\
\hline \multicolumn{11}{|c|}{ Age premier vêlage } \\
\hline (mois) & $38,4 \pm 0,8$ & $29,7 \pm 0,8$ & $34,6 \pm 0,7$ & $34,4 \pm 0,9$ & $33,2 \pm 0,6$ & $34,5 \pm 0,5$ & $33,5 \pm 1,0$ & $* * *$ & NS & NS \\
\hline \multicolumn{11}{|c|}{ Intervalle entre vêlages } \\
\hline (mois) & $21,8 \pm 0,5$ & $16,5 \pm 0,5$ & $17,8 \pm 0,5$ & $20,4 \pm 0,7^{a}$ & $19,3 \pm 0,5^{b}$ & $20,0 \pm 0,4 a, b$ & $18,3 \pm 0,7$ & $* * *$ & $*$ & $*$ \\
\hline
\end{tabular}

${ }^{1}$ Type $(\mathrm{L})$ local ou $(\mathrm{C})$ croisé avec des races laitières

${ }^{2}$ (A) complémentation absente, (S) simple minérale à base de sel gemme ou de bloc à lécher, ou (E) plus élaborée, à l'aide de concentrés protéo-énergétiques à base de tourteau d'arachide, de tourteau de palmiste, de son de riz ou de blé, associé au minéral

${ }^{3}$ (I) fourrage naturel ou (II) amélioré par cultures fourragères à base de Trypsacum laxum et de légumineuses fourragères

${ }^{\mathrm{a}, \mathrm{b}}$ Les moyennes intra-effets ne comportant pas de lettres communes sont significativement différentes au seuil de $5 \%$

de race locale vs $16,5 \pm 0,5$ mois chez les vaches de type croisé $(\mathrm{P}<0,001)$. La complémentation élaborée a permis un gain de 1,1 mois par rapport à la complémentation simple $(\mathrm{P}<0,05)$. L'absence de complémentation s'est caractérisée, de manière surprenante, par les valeurs les plus faibles (17,8 $\pm 0,5$ mois). De même, le fourrage amélioré a permis une réduction de l'intervalle entre vêlages de 1,7 mois par rapport au fourrage naturel $(\mathrm{P}<0,05)$.

Les effets d'interaction se sont tous révélés non significatifs, indépendamment de la variable étudiée. Les paramètres zootechniques (production laitière, âge au premier vêlage, intervalle entre vêlages, taux de fertilité, taux de mortalité) issus de la présente étude ont été comparés à ceux provenant de la littérature (tableau II).

\section{DISCUSSION}

La région d'étude est connue pour sa vocation agropastorale (26). La composition botanique des pâturages naturels indique que ces derniers se rapprochent de ceux du Burundi (11) et constituent une ressource naturelle non négligeable, qui peut être très productive si elle est bien gérée. Le fait que la majorité des éleveurs enquêtés pratiquaient essentiellement l'élevage traduit une forme de spécialisation des activités liées au sol, au moins en termes d'élevage ou d'agriculture. Il serait toutefois intéressant de sensibiliser les éleveurs pratiquant l'agriculture de subsistance à l'importance de conserver et de valoriser les résidus de récolte de manière rationnelle. Cette absence de valorisation contraste avec la pratique des cultures fourragères destinées à la supplémentation des animaux, ainsi qu'avec l'usage presque généralisé de la supplémentation à l'aide de blocs à lécher, du sel gemme et/ou de concentrés. La proportion élevée d'éleveurs enquêtés disposant d'un agent technique ex situ ou in situ, montre, d'autre part, qu'ils sont soucieux de la couverture sanitaire des animaux. Ces agents jouent vraisemblablement un rôle important dans l'ensemble des dispositions prises par les éleveurs, dont l'importance est liée au pouvoir d'achat.

Le taux de pénétration d'animaux croisés dans l'ensemble des exploitations a été proche de celui de 80 p. 100 rapporté en Ouganda (24). Ce fait associé à la pratique, même marginale, de l'insémination artificielle et au recours aux animaux reproducteurs par achat ou par confiage au sein des exploitations témoigne du fait que les éleveurs sont également sensibilisés aux notions d'amélioration génétique (12) et de rotation de la reproduction. Le faible pourcentage d'exploitations hébergeant exclusivement des animaux améliorés montre que la race locale reste très importante (7).

La structure des sex-ratios dans le présent échantillon était représentative de systèmes extensifs en zone tropicale avec un rapport femelles/mâles élevé, situation commune en élevage extensif avec une proportion de femelles qui se situe entre 70 et 75 p. 100 (27). Le rapport génisses / vaches adultes a montré une population animale en expansion, compte tenu du taux de réforme des vaches probablement très faible dans la région. La chute importante des effectifs dans les classes d'âge d'un à deux ans pouvait être attribuée à des problèmes de mortalité mais aussi à des sorties précoces de jeunes vendus pour subvenir aux besoins des éleveurs (27).

Le sevrage effectué à l'âge de huit mois dans la présente étude a été proche de celui observé dans les autres élevages de type traditionnel en pays tropicaux (21). Chez les animaux croisés, les meilleures productions laitières ont permis vraisemblablement une croissance plus rapide des jeunes et ainsi un sevrage plus précoce, ou un sevrage volontairement anticipé afin de bénéficier de la production laitière de l'animal. Il serait nécessaire de comparer les courbes de croissances des jeunes animaux pour étayer cette hypothèse.

Les taux de fertilité observés (52,5 p. 100 vs 41,3 p. 100, respectivement dans la race $\mathrm{L}$ et le type $\mathrm{C})$ ont été assez faibles $(19,22)$ et pouvaient être expliqués par le sevrage tardif des animaux. La faible valeur alimentaire des fourrages une partie de l'année en était également la cause, bien qu'elle n'ait pas été déterminée, ni d'éventuelles pathologies (Wanyoike, 2009, thèse non publiée). Les problèmes d'effectifs et d'accessibilité de taureaux conduisant à une surexploitation des mâles disponibles n'étaient pas à exclure non plus. Les performances particulièrement mauvaises des vaches croisées témoignaient d'une faible fertilité intrinsèque des femelles, liée à une sensibilité particulière aux conditions tropicales (5), ou à un taux de mortalité juvénile assez élevé des races exotiques (23), suggéré aussi par les présents résultats (tableau II). Les taux de mortalité apparaissent assez variables selon les auteurs, et plus particulièrement chez les animaux croisés. Cela peut résulter de différences de gestion des troupeaux ou vraisemblablement d'une plus grande sensibilité aux conditions environnementales prévalant dans la région (6). 


\section{Tableau II}

Tableau comparatif des différents paramètres zootechniques issus des résultats propres à cette étude et de données provenant de la littérature

\begin{tabular}{|c|c|c|c|c|}
\hline Paramètre & Race ou type & Valeur & Pays & Auteur \\
\hline \multirow[t]{7}{*}{ Production laitière (L/jour) } & Ankole & 2,4 & RD Congo & Présente étude \\
\hline & Ankole et Watutsi & 2,0 & Afrique centrale & Compère et Dupont, 2005 \\
\hline & Ankole & 2,4 & Ouganda & Galukande et coll., 2008 \\
\hline & Ankole & 2,75 & Burundi & Hatungumukama et coll., 2007 \\
\hline & Ankole $\mathrm{x}$ Frisonne & 5,2 & RD Congo & Présente étude \\
\hline & Ankole x Sahiwal (50-75 \%) & 3,69 & Burundi & Hatungumukama et coll., 2007 \\
\hline & Pure Holstein-Frisonne & 7,2 & Tanzanie & Bee et coll., 2006 \\
\hline \multirow[t]{7}{*}{ Age premier vêlage (mois) } & Ankole & 38,4 & RD Congo & Présente étude \\
\hline & Ankole et Watutsi & 30 et 36 & Afrique centrale & Compère et Dupont, 2005 \\
\hline & Ankole & 38,9 & Ouganda & Galukande, 2010 \\
\hline & Ankole & 33,2 & Ouganda & Kugonza et coll., 2011 \\
\hline & Ankole x Frisonne & 29,7 & RD Congo & Présente étude \\
\hline & Ankole x Frisonne (> $50 \%$ ) & 29,1 & Ouganda & Galukande G., 2010 \\
\hline & Zébu x Holstein-Frisonne & 34,7 & Ethiopie & Ibrahim et coll., 2011 \\
\hline \multirow[t]{9}{*}{ Intervalle vêlages (mois) } & Ankole & 21,8 & RD Congo & Présente étude \\
\hline & Ankole et Watutsi & 20 & Afrique centrale & Compère et Dupont, 2005 \\
\hline & Ankole & 12,9 & Ouganda & Kugonza et coll., 2011 \\
\hline & Ankole & 16,0 & Ouganda & Galukande, 2010 \\
\hline & Ankole x Frisonne & 16,5 & RD Congo & Présente étude \\
\hline & Zébu x Frisonne & 13,5 & Tanzanie & Bee et coll., 2006 \\
\hline & Sanga $x$ Holstein-Frisonne & 17 & Ghana & Obese et coll., 2009 \\
\hline & Ankole x Frisonne (> $50 \%$ ) & 15,3 & Ouganda & Galukande, 2010 \\
\hline & Holstein-Frisonne x zébu & 13,9 & Ethiopie & Ibrahim et coll., 2011 \\
\hline \multirow[t]{4}{*}{ Taux de fertilité (\%) } & Ankole & 52,5 & RD Congo & Présente étude \\
\hline & Ankole & 65 & Ouganda & Ndumu et coll., 2008 \\
\hline & Ankole x Frisonne & 41,3 & RD Congo & Présente étude \\
\hline & Zébu malgache x Sahiwal & $57-60$ & Sénégal & Mbay et Ndiay, 1991 \\
\hline \multirow[t]{16}{*}{ Taux de mortalité (\%) } & Ankole & 4,5 (0-1 an) & RD Congo & Présente étude \\
\hline & Ankole & 2,0 (1-2 ans) & RD Congo & Présente étude \\
\hline & Ankole & 5,4 (jusqu'à 1 an) & Ouganda & Kivaria et coll., 2004 \\
\hline & Ankole & 5 (avant sevrage) & Ouganda & Mulindwa et coll., 2010 \\
\hline & Ankole & 5,3 (génisses) & Ouganda & Mulindwa et coll., 2010 \\
\hline & Ankole & 4,2 (2-9 ans) & Ouganda & Mulindwa et coll., 2010 \\
\hline & Ankole $\mathrm{x}$ Frisonne & 6,4 (0-1 an) & RD Congo & Présente étude \\
\hline & Ankole $\mathrm{x}$ Frisonne & 4,2 (1-2 ans) & RD Congo & Présente étude \\
\hline & Ankole x Frisonne & 7,1 (avant sevrage) & Ouganda & Mulindwa et coll., 2010 \\
\hline & Ankole x Frisonne & 7,3 (génisses) & Ouganda & Mulindwa et coll., 2010 \\
\hline & Ankole $\mathrm{x}$ Frisonne & 6,5 (2-9 ans) & Ouganda & Mulindwa et coll., 2010 \\
\hline & Fogera x Frisonne (50 \%) & 8,3 (à 360 jours) & Ethiopie & Amuamuta et coll., 2006 \\
\hline & Fogera x Frisonne $(75 \%)$ & 16,0 (à 360 jours) & Ethiopie & Amuamuta et coll., 2006 \\
\hline & Races tropicales $x$ exotiques & 50 (avant 1 an) & Tanzanie & Said et coll., 2001 \\
\hline & N’Dama x Sahiwal & $6,7$ (0-6 mois $)$ & Ethiopie & Carew et coll., 1986 \\
\hline & N’Dama x Sahiwal & 16,6 (adultes) & Ethiopie & Carew et coll., 1986 \\
\hline
\end{tabular}


Hormis le lait, les 2,6 p. 100 d'animaux vendus par an ont servi vraisemblablement à couvrir les besoins de la famille. L'effectif des animaux vendus par an a montré d'ailleurs la place qu'occupait l'élevage dans la trésorerie des ménages. Néanmoins, par manque d'information sur le prix et la catégorie des animaux vendus, il n'a pas été possible d'évaluer ce que cette vente couvrait. Le pourcentage reste toutefois faible et signifie que les troupeaux étaient en croissance ou à effectifs stables si la croissance était compensée par un taux de mortalité équivalent. Toutefois, avec un effectif moyen de 133 animaux, 78 p. 100 de vaches et environ 47 p. 100 de fertilité, le nombre de veaux nés par exploitation était de l'ordre de 44. Compte tenu d'une mortalité cumulée des jeunes de $0-2$ ans de 8 p. 100 environ, le taux de mortalité en dehors de ces périodes d'âge aurait dû être de l'ordre de 30 p. 100 pour maintenir un effectif de troupeau stable. Ces données sont compatibles avec celles de la littérature (10). Elles pourraient expliquer pourquoi les mâles de plus de deux ans ont présenté de faibles effectifs. Mais il est possible que les éleveurs n'aient pas déclaré toutes les sorties des animaux liées aux ventes et aux dons.

La production laitière journalière moyenne de 2,4 L observée chez les vaches de race locale a été faible. Il s'agit d'une race non spécialisée mais bien adaptée aux conditions du milieu (6). Le niveau de croisement des animaux améliorés était inconnu et a été apprécié d'après leur phénotype. Ils ont atteint des productions moyennes de 5,2 L/jour, dans des conditions d'élevage rudimentaires. Ces valeurs sont restées faibles au regard du potentiel de la production des races améliorées dans leur milieu d'origine. Chez des animaux présentant 50 p. 100 de gènes Holstein, la production laitière observée est de 11,2 L/jour, bien qu'une production plus faible de 10,4 L/ jour soit enregistrée au-delà de cette proportion (8). A ce titre, il est remarquable de constater que les écarts entre les productions laitières observées dans les milieux tropicaux sont modestes, malgré des différences vraisemblables de potentiels génétiques et de conduite d'élevage (tableau II). Cela pourrait être dû à une loi des rendements décroissants lorsque le pourcentage de sang laitier augmente, réduisant proportionnellement la rusticité des animaux (6). Les vaches laitières dont le potentiel génétique maximal n'est pas exprimé répondent normalement bien à la complémentation à l'aide de concentrés (13). Dans cette étude, l'apport en concentré a eu une influence assez marginale sur la production laitière. Néanmoins, il faut signaler que les quantités distribuées n'ont pas été mesurées par les éleveurs et ont pu, de ce fait, être faibles. D'autre part, ces compléments ont été offerts uniquement après la mise bas, et indépendamment des niveaux de production et du stade de lactation. L'absence d'interaction suggère que l'effet positif du fourrage amélioré et de la complémentation protéo-énergétique est indépendant du niveau génétique et que même la race locale répond positivement à l'amélioration de l'alimentation (11).

Les différences d'âges au premier vêlage sont d'ordre physiologique, sanitaire ou de conduite de la mise à la reproduction. Dans cette étude, le fait que les animaux des deux types aient reçu en moyenne la même alimentation suggère un effet racial sur l'âge au premier vêlage, et donc des différences de précocité sexuelle. D’une façon générale, les éleveurs déclaraient une pratique de mise à la reproduction tardive de la race locale, afin d'éviter les risques de dystocie dus au faible développement de la cavité pelvienne de la jeune femelle. Il n'est toutefois pas exclu que, par manque des ressources financières, les éleveurs aient traité préférentiellement les animaux améliorés contre les maladies les plus courantes, comme rapporté en Ouganda (8), biaisant de ce fait les résultats.

Des différences entre types génétiques ont été observées pour les intervalles entre vêlages. Ce paramètre dépend de plusieurs éléments, tels que la durée d'allaitement du veau, la rapidité de la mise au taureau, les affections générales ou de l'appareil génital
(9), ou les déséquilibres nutritionnels (10), et probablement la race (2) mais aussi la difficulté de détection des chaleurs (20). Les valeurs observées restent relativement élevées mais sont couramment rencontrées en milieu tropical (tableau II). Les améliorations de pratiques d'élevage, dont l'alimentation, ont un effet positif sur les intervalles entre vêlages (5). Toutefois, paradoxalement et contrairement à l'effet du fourrage, les complémentations minérale et protéo-énergétique ont eu un effet négatif en allongeant cet intervalle. Cette observation est difficile à expliquer. Les éleveurs étaient peu sensibilisés à l'intérêt de complémenter les animaux avant le vêlage, les compléments ayant été de surcroît principalement distribués aux animaux pendant la lactation et en quantités non proportionnelles au niveau de la production laitière. Les éleveurs ne pesaient pas non plus régulièrement leurs animaux. Il reste donc difficile de préjuger des bilans énergétiques au cours de l'étude et de leur impact sur la reprise des cycles œstraux.

\section{CONCLUSION}

Cette étude menée sur 65 fermes d'élevage dans la région de Beni, au Nord Kivu, RDC, a montré que les éleveurs étaient relativement professionnalisés et spécialisés dans leur activité. Ils pratiquaient l'amélioration génétique des animaux, la complémentation alimentaire et la production fourragère, tout en étant encadrés par des agents techniques vétérinaires. Les taux de réforme des animaux étaient faibles, associés à des variations d'inventaire ou des mortalités élevées. De façon générale, les performances animales étaient faibles. L'amélioration génétique a augmenté sensiblement la production laitière et la fécondité des animaux mais également la mortalité juvénile. La complémentation alimentaire a eu peu d'effet sur ces paramètres, bien que des quantifications d'apports n'aient pu être effectuées. Néanmoins, des biais entre type génétique et gestion du troupeau existaient peut-être à ce niveau. L'amélioration génétique est une voie importante d'amélioration de la production laitière dans la région. Une évaluation plus précise des effets de l'alimentation sur des animaux de niveaux d'amélioration génétique différents doit cependant être poursuivie dans la région.

\section{Remerciements}

Les auteurs remercient la Coopération technique belge pour le financement de cette étude.

\section{BIBLIOGRAPHIE}

1. AMUAMUTA A., ASSEGED B., GOSHU G., 2006. Mortality analysis of Fogera calves and their Friesian crosses in Andassa cattle breeding and improvement ranch, Northwestern Ethiopia. Rev. Méd. vét., 157: 525-529.

2. BA DIAO M., DIENG A., SECK M.M., NGOMIBE R.C., 2006. Pratiques alimentaires et productivité des femelles laitières en zone périurbaine de Dakar. Rev. Elev. Méd. vét. Pays trop., 59 : 43-49.

3. BEE J.K.A., MSANGA Y.N., KAVANA P.Y., 2006. Lactation yield of crossbred dairy cattle under farmer management in Eastern Coast of Tanzania. Livest. Res. rural Dev., 18. http://www.Irrd.org//rrd18/2/ bee18023.htm (28/06/2012)

4. CAREW S.F., SANDFORD J., WISSOCQ Y.J., DURKIN J., TRAIL J.C.M., 1986. N'dama cattle productivity at Teko Livestock Station, Sierra Leone, and initial results from crossbreeding with Sahiwal. Addis Ababa, Ethiopia, ILCA. (Bull. No 23)

5. COMPERE R., DUPONT J., 2005. Elevage des bovins sur les hautes collines de I'Afrique centrale. In : Théwis A., Bourbouze R., Compère R., Duplan J.M., Hardouin J., Manuel de zootechnie comparée nord-sud. Paris, France, INRA, $637 \mathrm{p}$.

6. DEMEKER S., NESER F.W.C., SCHOEMAN J., 2004. Estimates of genetic parameters for Boran, Friesian and crosses of Friesian and Jersey with Boran cattle in tropical highlands of Ethiopia: milk production traits and cow weight. J. Anim. Breed. Genet., 121: 57-65. 
7. GALUKANDE G., 2010. Comparison of production systems with purebred Ankole vs crossbred Ankole-Friesian animals on-farm using a combined cross-sectional and longitudinal approach (Kiruhura District of Uganda). PhD Diss., University of Natural Resources and Applied Life Sciences, Vienna, Austria.

8. GALUKANDE $\mathrm{E}$, MULINDWA $\mathrm{H}$, WURZINGER $M$, OKEYO A.M., SOLKNER J., 2008. On-farm comparison of milk production and body condition of purebred Ankole and crossbred Friesian-Ankole cattle in South Western Uganda. In: Conf. Int. Res. Food Security, Natural Resource Management and Rural Development, University of Hohenheim, Tropentag, Uganda, 7-9 Oct. 2008. http://www.tropentag. de/2008/abstracts/full/62.pdf (5/03/2012

9. GAUTAM G, NAKAO T, YUSUF M, KOIKE K., 2009. Prevalence of endometritis during the postpartum period and its impact on subsequent reproductive performance in two Japanese dairy herds. Anim. Reprod. Sci., 116: 175-187.

10. GRIMAUD P., MPAIRWE D., CHALIMBAUD J., MESSAD S., FAYE B., 2007. The place of Sanga cattle in dairy production in Uganda. Trop. Anim. Health Prod., 39: 217-227.

11. HATUNGUMUKAMA G., HORNICK J.L., DETILLEUX J., 2007. Aspects zootechniques de l'élevage bovin laitier au Burundi : présent et futur. Ann. Méd. vét., 151 : 150-165.

12. HATUNGUMUKAMA G., HORNICK J.L., DETILLEUX J., 2009. Effects of non genetic and crossbreeding factors on daily milk yield of Jersey x Sahiwal x Ankole cows in Burundi. J. Anim. Vet. Adv., 8: 794798.

13. HATUNGUMUKAMA G., LEROY P.L., DETILLEUX J., 2008. Effects of non-genetic factors on daily milk yield of Friesian cows in Mahwa Station (South Burundi). Rev. Elev. Méd. vét. Pays trop., 61: 45-49.

14. HATUNGUMUKAMA G., SIDIKOU D.I., LEROY P.L., DETILLEUX I., 2007. Effects of non genetic and crossbreeding factors on daily milk yield of Ayrshire x (Sahiwal x Ankole) cows in Mahwa station (Burundi). Livest. Sci., 110: 111-117.

15. IBRAHIM N., ABRAHA A., MULUGETA S., 2011. Assessment of reproductive performances of crossbred dairy cattle (Holstein Friesian $\mathrm{x}$ zebu) in Gondar Town. Global Vet., 6: 561-566.

16. KIVARIA F.M., HEUER C., JONGEJAN F., OKELLO-ONEN J., RUTAGWENDA T., UNGER V., BOEHLE W., 2004. Endemic stability for Theileria parva infections in Ankole calves of the Ankole ranching scheme, Uganda. Onderstepoort J. vet. Res., 71: 189-95.

17. KUGONZA D.R., NABASIRYE M., MPAIRWE D., HANOTTE O., OKEYO A.M., 2011. Productivity and morphology of Ankole cattle in three livestock production systems in Uganda. Anim. Genet. Res., 48: 1322.

18. MARARO S.B., 2001. Pouvoirs, élevage bovin et la question foncière au Nord-Kivu. In : Reyntjens F., L'Afrique des Grands Lacs. Annuaire 2010-2011. Paris, France, L'Harmattan, 31 p.
19. MBAY E.M., NDIAY E.M., 1991. Etude des chaleurs, de la fertilité après un traitement de maîtrise de la reproduction chez la vache zébu Cobra. Dakar, Sénégal, Laboratoire national de l'élevage et de recherches vétérinaires, Institut sénégalais de recherches agricoles http://www. sist.sn/gsdl/collect/publi/index/assoc/HASH1a0d/bbf899e4.dir/doc.pdf $(9 / 10 / 2012)$

20. MELAKU M., ZELEKE M., GETINET M., MENGISTIE T., 2011. Reproductive performances of Fogera cattle at Metekel cattle breeding and multiplication ranch, North West Ethiopia. J. Anim. Feed Res., 1: 99-106.

21. MULINDWA H., GALUKANDE E., WURZINGER M., OKEYO A.M., SOLKNER J., 2010. Evaluation of Ankole pastoral production systems in Uganda: Systems analysis approach. http://www.eaap.org/Previous Annual_Meetings/2009Barcelona/Papers/27_Mulindwa.pdf (09/03/2012)

22. NDUMU D.B., BAUMUNG R., WURZINGER M., DRUCKER A.G., OKEYO A.M., SEMAMBO D., SOLKNER J., 2008. Performance and fitness traits versus phenotypic appearance in the African Ankole Longhorn cattle: A novel approach to identify selection criteria for indigenous breeds. Livest. Sci., 113: 234-24.

23. NGONGONI N.T., MAPIYE C., MWALE M., MUPETA B., 2007. Effect of supplementing a high-protein ram press sunflower cake concentrate on smallholder milk production in Zimbabwe. Trop. Anim. Health Prod., 39: 297-307. DOI: 10.1007/s11250-007-9018-0

24. NJARUI D.M.G. KABIRIZI J.M., ITABARI I.K., GATHERU M. NAKIGANDA A., MUGERWA S., 2012. Production characteristics and gender roles in dairy farming in peri-urban areas of Eastern and Central Africa. Livest. Res. rural Dev., 24. http://www.Irrd.org//rrd24/7/njar24122. htm $(13 / 12 / 2012)$

25. OBESE F.Y., DARFOUR-ODURO K.A., GOMDA Y., BEKOE E., 2009. Reproductive performance following artificial insemination in Sanga and crossbred (Friesian x Sanga) cows in the Accra plains of Ghana. In: Int Symp. Sustainable improvement of animal production and health, IAEA, Vienna, Austria, 8-11 June 2009. http://www-naweb.iaea.org/nafa/aph/ bookofextendedsynopses.pdf. (10/10/2012)

26. OCHA, 2005. Mission d'évaluation des besoins humanitaires dans la province du Nord-Kivu. Rapport 2005. République démocratique du Congo, OCHA $30 \mathrm{p}$

27. ONONO J.O., WIELAND B, RUSHTON J, 2012. Productivity in different cattle production systems in Kenya. Trop. Anim. Health Prod. DOI : $10.1007 / s 11250-012-0233-y$

28. SAID R., BRYANT M.J., MSECHU J.K.K, 2001. Growth and survival of crossbred beef cattle in Tanzania. Tanzanian Soc. Anim. Prod. 28: 126 134.

29. USMAN T., GUO G., SUHAIL S.M., AHMED S., QIAOXIANG L., QURESHI M.S., WANG Y., 2012. Performance traits study of Holstein Friesian cattle under subtropical conditions. J. Anim. Plant Sci., 22: 92-95.

Accepté le 27.02.2013 


\section{Summary}

Kibwana D.K., Makumyaviri A.M., Hornick J.L. Extensive farming practices and cattle performances of the local breed or crossed with exotic dairy breeds in the Democratic Republic of Congo

In order to analyze the relationship between farming practices and animal performances of mixed (dairy and beef) breed cows in Beni area, North Kivu Province, Democratic Republic of Congo, a study was conducted from July 2003 to November 2006. The sample consisted of 8676 cattle head from 65 farms, among which 4945 cows were of local breed, others were crossed with exogenous breeds considered ameliorative. Surveys, demographic observations, milk production measurements, and reproduction monitoring were carried out in the farms of the study. Data were analyzed with the chi-squared test and generalized linear models according to the genotype, forage quality and supplement characteristics. Results showed some level of technical proficiency in farmers, but a low turnover rate of the herds. Breeding practices impacted on milk production, age at first calving and calving interval. In crossed-type cows, milk production was more than twice that observed in the local breed. Supplementation with forage and concentrates significantly, but also little increased milk production compared to no supplementation. The age at first calving and calving interval were influenced by the genotype, and the calving interval was influenced by the supplementation and forage type. These results highlight the effects of livestock management on milk and reproductive performances of cows in the region of the study.

Keywords: Cattle - Livestock breed - Crossbreeding - Animal performance - Animal husbandry method - Democratic Republic of Congo.

\section{Resumen}

Kibwana D.K., Makumyaviri A.M., Hornick J.L. Prácticas de cría extensiva y de rendimientos bovinos de la raza local, y cruces de razas lecheras exóticas en Beni, República democrática de Congo

Con el fin de analizar las prácticas de cría y su relación con los rendimientos zootécnicos de bovinos de raza mixta, leche y carne, en el territorio de Beni, provincia de Nor-Kivu, República democrática de Congo, se llevó a cabo un estudio entre julio 2003 y noviembre 2006, en 8676 bovinos provenientes de 65 criaderos y comportando 4945 vacas de raza local y cruzadas con razas exógenas consideradas mejoradoras. En las explotaciones incluidas en el estudio, se llevaron a cabo encuestas, observaciones demográficas, medidas de producción lechera y seguimientos de reproducción. Los datos fueron analizados con la ayuda del test de Chi cuadrado, y de modelos lineares generalizados en función del tipo genético, de la calidad del forraje y del tipo de suplemento. Los resultados muestran un cierto nivel de tecnicidad por parte de los criadores, una baja renovación de las poblaciones bovinas y una influencia de las prácticas de cría sobre la producción lechera, la edad al primer parto y el intervalo entre partos. En las vacas de tipo cruzado, la producción de leche diaria fue más del doble de la observadas en la raza local. La suplementación bajo la forma de forrajes y de concentrados permitió aumentar significativamente, pero en forma frágil la producción lechera con respecto a la situación sin complemento. La edad al primer parto y el intervalo entre partos fueron influenciados por el tipo genético y el intervalo entre partos igualmente por la suplementación y el tipo de forraje. Estos resultados muestran la importancia del comportamiento de los criaderos con respecto a los rendimientos lecheros y reproductivos de las vacas de la región estudiada.

Palabras clave: Ganado bovino - Raza de ganado Cruzamiento - Desempeño animal - Metodo de crianza República Democrática del Congo. 